\title{
Eigenanalysis interference cancellers with robust capabilities ${ }^{1}$
}

\author{
Cheng-Chou Lee, Ju-Hong Lee* \\ Department of Electrical Engineering, Room 517, 2nd Building, National Taiwan University, Taipei, 106, Taiwan
}

Received 11 October 1995; revised 6 May 1996 and 6 January 1997

\begin{abstract}
The maximum rejection of interference in the presence of steering angle error for adaptive array processing is considered. It has been shown that eigenanalysis interference cancellers (EICs) possess the advantage of providing maximal suppression of undesired interference with fast convergence over conventional adaptive beamformers. However, the performance of EICs is also very sensitive to steering angle error when the number of signal sources is overestimated. We present an artificial source injection technique to make EICs have robust capabilities against steering angle error. Modifications for enhancing the proposed technique are also presented. It is shown that the proposed technique can alleviate the drawback like the loss of degrees of freedom when using existing EICs. Simulation examples are provided for illustrating the effectiveness of the proposed technique. (O) 1997 Elsevier Science B.V.
\end{abstract}

\section{Zusammenfassung}

Es wird die maximale Interferenzrückweisung für adaptive Antennengruppen bei Vorhandensein eines Richtwinkelfehlers betrachtet. Es ist gezeigt worden, daß Interferenzlöscher auf der Basis einer Eigenanalyse (EIC) gegenüber konventionellen adaptiven Keulenformern den Vorzug aufweisen, die maximale Unterdrückung der unerwünschten Interferenz mit schneller Konvergenz zu bewerkstelligen. Die Leistungsfähigkeit der EICs ist jedoch auch sehr empfindlich auf Richtwinkelfehler, wenn die Anzahl der Signalquellen überschätzt wird. Wir stellen ein Verfahren zum Einsatz künstlicher Quellen vor, um EICs mit robusterem Verhalten gegenüber Richtwinkelfehlern auszustatten. Modifikationen zur weiteren Verbesserung des vorgeschlagenen Verfahrens werden auch vorgestellt. Es wird gezeigt, wie das vorgeschlagene Verfahren Nachteile wie den Verlust an Freiheitsgraden beim Einsatz existierender EICs vermindern kann. Simulationsbeispiele sind zur Illustration der Wirksamkeit des vorgeschlagenen Verfahrens beigeschlossen. (O) 1997 Elsevier Science B.V.

\section{Résumé}

Le rejet maximum d'interférence en présence d'erreurs sur l'angle directeur est étudié dans le cas du traitement adaptatif de matrices. On sait que les "éliminateurs d'interférence d'analyse de valeurs propres" (EICs pour Eigenanalysis Interference Cancellers) possèdent l'avantage de fournir une suppression maximale d'interférences indésirables avec une convergence rapide par rapport aux tcchniques conventionnelles de formation adaptative de faisceaux. Cependant, les performances des EICs sont également très sensibles aux erreurs sur l'angle directeur lorsque le nombre de sources des signaux est surestimé. Nous présentons ici une technique d'injection artificielle de source afin de rendre les EICs robustes face aux erreurs sur l'angle directeur, ainsi que certaines modifications en vue d'améliorer la technique proposée. On montre aussi que cette dernière

\footnotetext{
${ }^{1}$ Supported by the National Science Council under Grant NSC85-2213-E002-008.

* Corresponding author. Fax: +88623671909.
} 
peut atténuer certains inconvénients telle la perte de degrés de liberté lorsqu'on utilise des EICs existants. Des exemples de simulation sont fournis pour illustrer l'efficacité de la technique proposée. (C) 1997 Elsevier Science B.V.

\section{Introduction}

Adaptive interference cancellation is usually required for maximizing the rejection of interference regardless of the interference-to-noise power ratio (INR) when processing array data. Techniques for achieving this purpose by using adaptive interference cancellers have been reported in [1-5,7]. Notable among them is the one of [4] where an eigenanalysis interference canceller (EIC) with fast convergence speed using a uniform linear array (ULA) was presented. The optimal weight vector is computed by minimizing the output noise power subject to a constraint of orthogonality to the interference subspace (IS). The IS is obtained through the generalized eigenvalue decomposition (GEVD) of the correlation matrix of the data vector at an appropriately designed blocking processor which blocks the desired signal from the received data vector.

In general, the number of interferers must be known a priori in order to make sure that the IS is spanned by the generalized eigenvectors which are obtained from GEVD. For practical circumstances, we have to estimate the number of interferers since the information regarding the number of interferers is usually not available. A useful method based on the information theoretic criteria AIC and MDL has been proposed in [8] for estimating the number of signal sources. However, it has been shown in [8] that the estimated number may be greater than the actual number. Fortunately, overestimation may be required under nonstationary situations where some smart interferers perhaps appear suddenly. Moreover, we can easily show that it causes only the increment of output noise power but still provides nulls at all interferers if the steering angle of the desired signal is correct during the implementation of the EIC. However, the desired signal cannot be eliminated completely by the blocking processor when the steering angle is not correct. As a result, overestimation of the number of the interferers leads to that the direction vector of the desired signal lies in the resulting IS due to the leakage of the desired signal in the blocked data vector. Therefore, signal cancellation arises because of the orthogonality between the resulting optimal weight vector and the IS.

To solve the above problem, we present an artificial source injection (ASI) technique in this paper. This technique tends to prevent the response vector of the desired signal from lying in the resulting IS by imposing artificial signal sources. The criteria required for determining the characteristics including the number, the direction angles, and the powers of the artificial signal sources are presented. Moreover, modifications for enhancing the proposed technique are also presented to alleviate the drawback like the loss of degrees of freedom by using the shift-invariant property of a ULA. It is shown that the proposed ASI technique makes the EIC possess robust capabilities against steering angle error.

This paper is organized as follows. Section 2 briefly describes the principle of the EIC and the problem in the presence of steering angle error. We present the ASI technique in Section 3. The criteria for determining the number, the direction angles, and the powers of the artificial signal sources are described in detail. Section 4 presents the modifications for enhancing the proposed technique under some considered situations. Simulation examples for showing the effectiveness of the proposed technique are presented in Section 5. We finally conclude this paper in Section 6 .

\section{The eigenanalysis interference cancellers}

Consider an $M$-sensor linear array with interelement spacing equal to $d$ illuminated by $P$ narrow-band signal sources from the distinct direction angles $\theta_{i}$, $i=1,2, \ldots, P$. Let the response of the $m$ th sensor to a signal with unit amplitude and a direction angle $\theta$ be given by $a_{m}(\theta)=\exp (\mathrm{j} 2 \pi(m-1) d \sin (\theta) / \lambda)$, where $\lambda$ is the wavelength of the signal sources. The received signal at the $m$ th sensor can be expressed as

$x_{m}(t)=\sum_{i=1}^{P} s_{i}(t) a_{m}\left(\theta_{i}\right)+n_{m}(t)$, 
where $s_{i}(t)$ denotes the complex amplitude of the $i$ th signal impinging on the array with direction angle $\theta_{i}$, and $n_{m}(t)$ the noise received by the $m$ th sensor. Both the signal and sensor noise are assumed to be independent and zero-mean stationary Gaussian random processes. In vector form, the received data vector is given by

$X(t)=\sum_{i=1}^{P} A\left(\theta_{i}\right) S_{i}(t)+N(t)=A_{s} S(t)+N(t)$,

where the response vector of the $i$ th signal is $A\left(\theta_{i}\right)=\left[a_{1}\left(\theta_{i}\right), a_{2}\left(\theta_{i}\right), \ldots, a_{M}\left(\theta_{i}\right)\right]^{\mathrm{T}}$, the noise vector is $N(t)=\left[n_{1}(t), n_{2}(t), \ldots, n_{M}(t)\right]^{\mathrm{T}}$, the signal source vector is $S(t)=\left[s_{1}(t), s_{2}(t), \ldots, s_{P}(t)\right]^{\mathrm{T}}$, and the response matrix of the signal sources is $\boldsymbol{A}_{s}=\left[A\left(\theta_{1}\right), A\left(\theta_{2}\right), \ldots, A\left(\theta_{P}\right)\right]$. The superscript $\mathrm{T}$ denotes the transpose operation.

Assume that the direction angle of the desired signal is $\theta_{1}$ and the steering vector with the steering angle $\theta_{0}$ is denoted by $A\left(\theta_{0}\right)=\left[a_{1}\left(\theta_{0}\right), a_{2}\left(\theta_{0}\right), \ldots, a_{M}\left(\theta_{0}\right)\right]^{\mathrm{T}}$, where $a_{m}\left(\theta_{0}\right)=\exp \left(\mathrm{j} 2 \pi(m-1) d \sin \left(\theta_{0}\right) / \lambda\right)$ for $m=$ $1,2, \ldots, M$. Based on the principle of the EIC presented in [4], a blocking matrix $\boldsymbol{B}$ is appropriately designed and used as the block processor such that $\boldsymbol{B}^{\mathrm{H}} A\left(\theta_{0}\right)=0$ in order to block the desired signal from the received data vector, where the superscript $\mathrm{H}$ denotes the complex conjugate and transpose operation. Let the order of $\boldsymbol{B}$ be $q$ and an $M \times 1$ vector $\boldsymbol{b}=\left[b_{0}, b_{1}, \ldots, b_{q}, 0, \ldots, 0\right]^{\mathrm{T}}$ with the nonzero entries satisfying the following relationship:

$\left(z-\mathrm{e}^{\mathrm{j} 2 \pi d \sin \left(\theta_{0}\right) / i}\right)^{q}=\sum_{k=0}^{q} b_{k}^{*} z^{k}$,

where the subscript ' $*$ ' denotes the complex conjugate. Thus, $\boldsymbol{B}$ can be expressed as

$\boldsymbol{B}=\left[\boldsymbol{b}, \tilde{\boldsymbol{I}}_{M} \boldsymbol{b}, \tilde{\boldsymbol{I}}_{M}^{2} \boldsymbol{b}, \ldots, \tilde{\boldsymbol{I}}_{M}^{M-q-1} \boldsymbol{b}\right]$,

where $\tilde{\boldsymbol{I}}_{M}=\left[\boldsymbol{u}_{2}, \boldsymbol{u}_{3}, \ldots, \boldsymbol{u}_{M}, \boldsymbol{u}_{1}\right]$ represents a cyclicshifting matrix with element $\boldsymbol{u}_{i}$ given by the $i$ th column vector of an $M \times M$ identity matrix. For simplicity, let $\theta_{0}=0^{\circ}$. Without the steering angle error, i.e., $\theta_{1}=\theta_{0}$, the blocked data vector is given by

$$
\begin{aligned}
\boldsymbol{B}^{\mathrm{H}} X(t) & =\sum_{i=2}^{P} \bar{A}\left(\theta_{i}\right) \bar{s}_{i}(t)+\boldsymbol{B}^{\mathrm{H}} N(t) \\
& =\overline{\boldsymbol{A}}_{j} S_{j}(t)+\boldsymbol{B}^{\mathrm{H}} N(t),
\end{aligned}
$$

where $\bar{A}\left(\theta_{i}\right)=\left[a_{1}\left(\theta_{i}\right), a_{2}\left(\theta_{i}\right), \ldots, a_{M-q}\left(\theta_{i}\right)\right]^{\mathrm{T}}, S_{j}(t)=$ $\left[\bar{s}_{2}(t), \bar{s}_{3}(t), \ldots, \bar{s}_{P}(t)\right]^{\mathrm{T}}, \bar{A}_{j}=\left[\bar{A}\left(\theta_{2}\right), \bar{A}\left(\theta_{3}\right), \ldots, \bar{A}\left(\theta_{P}\right)\right]$, and $\bar{s}_{i}(t)=v_{i} s_{i}(t)$ with $v_{i}=\left(\exp \left(\mathrm{j} 2 \pi d \sin \left(\theta_{i}\right) / \lambda\right)-1\right)^{q}$ representing the effect of the blocking processing on the $i$ th signal. The ensemble correlation matrix of $\boldsymbol{B}^{\mathrm{H}} X(t)$ is given by

$$
\begin{aligned}
\overline{\boldsymbol{R}} & =E\left\{\boldsymbol{B}^{\mathrm{H}} X(t) X^{\mathrm{H}}(t) \boldsymbol{B}\right\} \\
& =\overline{\boldsymbol{A}}_{j} \Psi_{j} \overline{\boldsymbol{A}}_{j}^{\mathrm{H}}+\pi_{n} \boldsymbol{B}^{\mathrm{H}} \boldsymbol{B},
\end{aligned}
$$

where $\Psi_{j}=E\left\{S_{j}(t) S_{j}^{\mathrm{H}}(t)\right\}$ and $\pi_{n}$ denotes the power of the spatially white sensor noise. After performing the GEVD on (6), we have the following relationship for the resulting generalized eigenvectors ( $g$-vectors) $g_{i}$ and generalized eigenvalues ( $g$-values) $\lambda_{i}$ :

$\overline{\boldsymbol{R}} g_{i}=\lambda_{i} \boldsymbol{B}^{\mathrm{H}} \boldsymbol{B} g_{i}$

where $\lambda_{1} \geqslant \lambda_{2} \geqslant \cdots \geqslant \lambda_{P-1}>\lambda_{P}=\cdots=\lambda_{M-q}-\pi_{n}$. Let $J$ be the estimated dimension of the IS. Using the $g$-vectors $g_{i}$, we construct a matrix as follows:

$\boldsymbol{G}=\left[g_{1}, g_{2}, \ldots, g_{J}\right]$.

Then, it is easy to show that the matrix $\bar{A}_{j}$ spans the same subspace as the matrix $\boldsymbol{B}^{\mathrm{H}} \boldsymbol{B} \boldsymbol{G}$, i.e., range $\left\{\bar{A}_{j}\right\}=\operatorname{range}\left\{\boldsymbol{B}^{\mathrm{H}} \boldsymbol{B} \boldsymbol{G}\right\}$ if the estimated number of the interferers is correct, i.e., $J=P-1$. The optimal weight vector can be obtained by solving the following optimization problem [4]:

Minimize $\bar{W}^{\mathrm{H}} \bar{W}$

$$
\text { subject to } \bar{W}^{\mathrm{H}} \bar{E}_{j}=0 \text { and } \bar{W}^{\mathrm{H}} \bar{A}\left(\theta_{1}\right)=1 \text {, }
$$

where $\overline{\boldsymbol{E}}_{j}=\boldsymbol{B}^{\mathrm{H}} \boldsymbol{B} \boldsymbol{G}$. We can show that the optimal weight vector for (9) is given by

$\overline{\boldsymbol{W}}_{0}=\kappa\left(\boldsymbol{I}-\overline{\boldsymbol{E}}_{j}\left(\overline{\boldsymbol{E}}_{j}^{\mathrm{H}} \overline{\boldsymbol{E}}_{j}\right)^{-1} \overline{\boldsymbol{E}}_{j}^{\mathrm{H}}\right) \bar{A}\left(0_{1}\right)$,

where $\kappa$ represents the corresponding normalization constant.

On the other hand, if the number of the interferers is overestimated, i.e., $J>P-1$, then $J-P+1$ more $g$ vectors are contained in the matrix $G$ of (8). It follows that range $\left\{\overline{\boldsymbol{A}}_{j}\right\} \subseteq$ range $\left\{\overline{\boldsymbol{E}}_{j}\right\}$. Consequently, the array performance suffers degradation since the dimension of the resulting constraints is increased, though the array with the optimal weight vector computed from (10) still provides nulls in the interference directions. 
Next, we consider the practical circumstances where the steering angle $\theta_{0}$ may differ from the direction angle $\theta_{1}$ of the desired signal. In this case, the blocking matrix $B$ which is designed such that $\boldsymbol{B}^{\mathrm{H}} A\left(\theta_{0}\right)=0$ yields a nonzero $\boldsymbol{B}^{\mathrm{H}} A\left(\theta_{1}\right)$. Therefore, the blocked data vector becomes

$$
\begin{aligned}
\boldsymbol{B}^{\mathrm{H}} X(t) & =\sum_{i=1}^{P} \bar{A}\left(\theta_{i}\right) \bar{s}_{i}(t)+\boldsymbol{B}^{\mathrm{H}} N(t) \\
& =\overline{\boldsymbol{A}}_{s} S_{s}(t)+\boldsymbol{B}^{\mathrm{H}} N(t),
\end{aligned}
$$

where $\overline{\boldsymbol{A}}_{s}=\left[\bar{A}\left(\theta_{1}\right), \bar{A}_{j}\right]$ and $S_{s}(t)=\left[\bar{s}_{1}(t), S_{j}^{\mathrm{T}}(t)\right]^{\mathrm{T}}$. The ensemble correlation matrix of $\boldsymbol{B}^{\mathrm{H}} X(t)$ is given by

$\overline{\boldsymbol{R}}=E\left\{\boldsymbol{B}^{\mathrm{H}} X(t) X^{\mathrm{H}}(t) \boldsymbol{B}\right\}=\bar{A}_{s} \Psi_{s} \bar{A}_{s}^{\mathrm{H}}+\pi_{n} \boldsymbol{B}^{\mathrm{H}} \boldsymbol{B}$,

where $\Psi_{s}=E\left\{S_{s}(t) S_{s}^{\mathrm{H}}(t)\right\}$. Eq. (12) reveals that the correlation matrix always contains the leakage component due to the desired signal. As a result, performing the GEVD on (12) usually generates $P$ principal $g$-values which are greater than $\pi_{n}$. In this case, we have range $\left\{\bar{A}_{s}\right\} \subseteq$ range $\left\{\bar{E}_{j}\right\}$ if the number of the interferers is overestimated. This is because $J>P-1$ and $J-P+1$ more $g$-vectors are contained in the matrix $G$ which is constructed from (8). Accordingly, the optimal weight vector given by

$\bar{W}_{0}=\kappa\left(\boldsymbol{I}-\overline{\boldsymbol{E}}_{j}\left(\overline{\boldsymbol{E}}_{j}^{\mathrm{H}} \overline{\boldsymbol{E}}_{j}\right)^{-1} \overline{\boldsymbol{E}}_{j}^{\mathrm{H}}\right) \vec{A}\left(\theta_{0}\right)$

will generate zero gain at the interferers as well as the desired signal. Therefore, the EIC fails completely regardless of the input signal to noise ratio (SNR) if the number of the interferers is overestimated in the presence of steering angle error.

\section{The proposed technique}

From the above section, it is noted that eliminating the component of the response vector corresponding to the desired signal from the estimated IS can avoid the desired signal cancellation in the presence of steering angle error and overestimation of the interference number. To achieve this goal, we construct a correlation matrix based on artificial source injection as follows:

$$
\overline{\boldsymbol{R}}_{a}=\overline{\boldsymbol{R}}+\sum_{k=1}^{h} \varepsilon_{k} \bar{A}\left(\Theta_{k}\right) \bar{A}^{\mathrm{H}}\left(\Theta_{k}\right),
$$

where $\bar{R}$ is given by (12) and $h$ denotes the number of the injected sources. $\varepsilon_{k}$ and $\Theta_{k}$ are the power and the direction angle of the $k$ th injected source, respectively. Let the difference between each of the direction angles of the interferers and the steering angle be not very small. Moreover, the steering angle error is small such that $\left|v_{1}\right|=\left|\left(\exp \left(\mathrm{j} 2 \pi d \sin \left(\theta_{1}\right) / \lambda\right)-1\right)^{q}\right| \ll 1$. In the following, we present the required criteria for choosing the appropriate values for $h, \varepsilon_{k}$ and $\Theta_{k}$.

\subsection{The number of the injected sources}

It is well known that the performance of the EIC suffers serious degradation when the dimension of the estimated IS is less than the number of the interferers since some of the interferers cannot be suppressed. The number of interferers impinging on the array must be estimated in order to determine how many artificial sources should be injected. Conventionally, estimation of the number of the interferers is performed based on the received data vector $X(t)$. Many methods have been presented in the literature for estimating the source number of $X(t)$. Notable among them are those based on the information theoretic criteria like AIC and MDL detection methods presented in [8]. However, the estimated number is a random variable which may not equal the actual number of the interferers. One way to deal with this difficulty is to find the error probabilities as presented in [9] when employing the AIC and MDL methods and then compute a satisfactory confidence interval for the number of interferers. Accordingly, the range for the actual number of interferers can be determined based on a desired confidence interval. Consequently, the actual number of interferers will be outside this range with a very small probability. However, how to compute the related probabilities and the range depends on the detection method used and is beyond the scope of this paper. Therefore, we only assume that the range for the actual number of interferers is already computed and then determine the required number of the injected sources.

Assume that the estimated number of the interferers is $\tilde{J}$ by using the AIC or MDL criteria proposed in [8] and the actual number of the interferers is in the range of $\left[\tilde{J}-\Delta_{1}, \tilde{J}+\Delta_{2}\right]$. Therefore, the dimension of the estimated IS must be equal to $J$ given by $\tilde{J}+\Delta_{2}$ in order to prevent the situation of underestimating the 
number of the interferers. On the other hand, the actual number of the interferers may equal to $\tilde{J}-\Delta_{1}$. Hence, there are at most $\Delta_{1}+\Delta_{2}$ in difference between the estimated and the actual dimensions of the IS. Based on the above discussion, we note that the number of sources injected must be at least equal to $h=\Delta_{1}+\Delta_{2}$.

\subsection{The input powers of the injected sources}

From (11), the power of the $i$ th signal source at the output of the blocking matrix is given by

$\bar{\pi}_{i}=E\left\{\left|\bar{s}_{i}(t)\right|^{2}\right\}=\left|v_{i}\right|^{2} \pi_{i}$,

where $\bar{s}_{i}(t)=v_{i} s_{i}(t)$ with $v_{i}=\left(\exp \left(\mathrm{j} 2 \pi d \sin \left(\theta_{i}\right) / \lambda\right)-\right.$ $1)^{q}$ and $\pi_{i}=E\left\{\left|s_{i}(t)\right|^{2}\right\}$ denotes the input power of the $i$ th signal source. For the case of small steering angle error with $\left|v_{1}\right| \ll\left|v_{i}\right|$ and $\bar{\pi}_{1} \ll \bar{\pi}_{i}$ for $i=2,3, \ldots, P$, it is appropriate to set the power for the $k$ th injected source as follows:

$\bar{\pi}_{1} \ll \varepsilon_{k} \ll \bar{\pi}_{i}$

for $i=2,3, \ldots, P$ and $k=1,2, \ldots, h$. The first inequality of (16) is required to guarantee that the component of the desired signal lying in the estimated IS can be reduced sufficiently, whilc the sccond inequality is employed to alleviate the deviation between the actual IS and the estimated IS due to these injected sources. For small steering angle error, we have the following approximation:

$$
\begin{aligned}
\left|v_{1}\right|^{2} & =\left|\exp \left(\mathrm{j} 2 \pi d \sin \left(\theta_{1}\right) / \lambda\right)-1\right|^{2 q} \\
& \approx\left(2 \pi d \sin \left(\theta_{1}\right) / \lambda\right)^{2 q},
\end{aligned}
$$

which decreases as $q$ increases. Hence, a signal blocking matrix $\boldsymbol{B}$ with higher order can be used to ensure the inequalities shown in (16) when the desired signal power is large.

In practice, $\bar{\pi}_{i}$ for $i=1,2, \ldots, P$ in (16) are unavailable. Moreover, it is even impossible to obtain accurate estimates for $\bar{\pi}_{i}$ because the direction angles of the signal sources are not known. However, if we can find the possible maximum of $\bar{\pi}_{1}$ and the possible minimum of $\bar{\pi}_{i}$ for $i=2,3, \ldots, P$, designated by $\bar{\pi}_{\mathrm{d}}$ and $\bar{\pi}_{\mathrm{u}}$, respectively, then (16) can still be guaranteed by setting such $\varepsilon_{k}$ that $\bar{\pi}_{\mathrm{d}} \ll \varepsilon_{k} \ll \bar{\pi}_{\mathrm{u}}$. In the following, we present an appropriate scheme for finding $\bar{\pi}_{\mathrm{d}}$ and $\bar{\pi}_{\mathrm{u}}$. Let the possible direction angles of the desired signal and interferers be in the ranges of $\left|\theta_{1}-\theta_{0}\right| \leqslant \delta \theta_{\mathrm{d}}$ and $\left|\theta_{i}-\theta_{0}\right| \geqslant \delta \theta_{\mathrm{u}}$ for $i=2,3, \ldots, P$, respectively, where $\delta \theta_{\mathrm{d}}<\delta \theta_{\mathrm{u}}$. Again, let $\theta_{0}=0$ for simplicity. Then, the proposed scheme is summarized as follows.

Step 1. From the eigenvalue decomposition (EVD) of the correlation matrix $\boldsymbol{R}$ of $X(t)$ which has been performed in Section 3.1 when utilizing the AIC or MDL criteria, find the corresponding eigenvalues $\tau_{i}$ in descending order for $i=1,2, \ldots, M$.

Step 2. From Section 3.1, set the estimated number of signal sources to $\tilde{J}+1$ and take $\pi_{\mathrm{u}}=\tau_{\tilde{J}+1}-\pi_{n}$ as the minimum signal power. If $\pi_{n}$ is also not available, it can be set to the average of the smallest $M-\tilde{J}-1$ eigenvalues.

Step 3. Based on the fact that $|\exp (\mathrm{j} 2 \pi d \sin (\theta) / \lambda)-1|$ monotonically increases as $|\theta|$ increases for $d=\lambda / 2$ and $|\theta| \leqslant \pi / 2$, we compute the minimum interference power $\bar{\pi}_{\mathrm{u}}$ after the blocking matrix $B$ as follows:

$\bar{\pi}_{\mathrm{u}}=\pi_{\mathrm{u}}\left|\exp \left(\mathrm{j} 2 \pi d \sin \left(\delta \theta_{\mathrm{u}}\right) / \lambda\right)-1\right|^{2 q}$.

Step 4. To estimate $\bar{\pi}_{\mathrm{d}}$, we first utilize a conventional non-adaptive beamformer which is pointing to $\theta_{0}=0$ with sufficiently low sidelobes and unit array gain at the direction angle $\theta_{0}=0$. Let the received power be $\pi_{\mathrm{c}}$. Then, we compute $\bar{\pi}_{\mathrm{d}}$ as follows:

$\bar{\pi}_{\mathrm{d}}=\pi_{\mathrm{c}} g_{\mathrm{c}}^{-2}\left|\exp \left(\mathrm{j} 2 \pi d \sin \left(\delta \theta_{\mathrm{d}}\right) / \lambda\right)-1\right|^{2 q}$,

where $g_{\mathrm{c}}$ is the array gain of the non-adaptive beamformer at the direction angle $\theta_{0}+\delta \theta_{\mathrm{d}}=\delta \theta_{\mathrm{d}}$.

Step 5. Determine the appropriate blocking order $q$ such that $\bar{\pi}_{\mathrm{d}} \ll \bar{\pi}_{\mathrm{u}}$.

Step 6. Select the appropriate injected source power $\varepsilon_{k}$ such that $\bar{\pi}_{\mathrm{d}} \ll \varepsilon_{k} \ll \bar{\pi}_{\mathrm{u}}$ is satisfied.

\subsection{The direction angles of the injected sources}

From the optimization problem formulated in (9) and its optimal solution given by (10), we note that the optimal weight vector which minimizes the output noise power is orthogonal to all of the vectors lying in the estimated IS. Moreover, the degrees of freedom in finding the optimal weight vector decrease and hence the output noise power increases as the overestimation 
of the number of the interferers increases even if the steering angle error does not exist. Therefore, the next problem is to find the appropriate direction angles of the injected sources after the appropriate number of the injected sources is decided. For simplicity, let the interelement spacing $d$ be $\lambda / 2$.

For an array with $M$ sensors, the $M$ nulls associated with its quiescent beam pattern are located at the angles $\theta$ which satisfy $A^{\mathrm{H}}\left(\theta_{0}\right) A(\theta)=0$, i.e., $\theta=\arcsin (2 k / M), \quad$ for $\quad k=-\langle M / 2\rangle, \ldots,-1,1, \ldots$, $\langle M / 2\rangle$, where $\langle x\rangle$ denotes the largest integer not greater than $x$. In the presence of $J$ interferers, the resulting optimal weight vector adjusts the $J$ nulls nearest to the $J$ interferers such that the array provides $J$ nulls for suppressing the $J$ interferers. In practice, the number $M$ of array sensors is much greater than the number $J$ of the interferers. The locations of the other $M-J$ nulls of the beam pattern will be almost the same as those of the quicscent beam pattern without interference. To reduce the possible distortion of the beam pattern due to artificial source injection, the angles of these $M-J$ nulls can then be used as the direction angles of the injected sources.

For the EIC employing a blocking matrix given by (4), the available degrees of freedom become $M-q$. To determine the angles corresponding to these $M-$ $q-J$ nulls from the $M-q$ nulls of the quiescent beam pattern, a simple approach is presented as follows.

Step 1. Construct the direction vectors $\bar{A}\left(\alpha_{k}\right)$ with the direction angles $\alpha_{k}=\arcsin (2 k /(M-q))$, for $k=$ $-\langle(M-q) / 2\rangle,-\langle(M-q) / 2\rangle+1, \ldots,-1,1,2, \ldots$ $\langle(M-q) / 2\rangle$, corresponding to the nulls of the quiescent beam pattern of an array with an equivalent size $M-q$.

Step 2. Compute the projection of each $\bar{A}\left(\alpha_{k}\right)$ onto the correlation matrix $\tilde{\boldsymbol{R}}$ corresponding to the data vector $\left[x_{1}(t), x_{2}(t), \ldots, x_{M-q}(t)\right]^{\mathrm{T}}$ as follows:

Projection $\left\{\bar{A}\left(\alpha_{k}\right)\right\}=\bar{A}^{\mathrm{H}}\left(\alpha_{k}\right) \tilde{\boldsymbol{R}} \bar{A}\left(\alpha_{k}\right)$.

Step 3. Choose the $\Delta_{1}+\Delta_{2}$ smallest projections from all of the projections computed from (20) and their corresponding direction vectors.

Step 4. Take the direction angles associated with these $\Delta_{1}+\Delta_{2}$ direction vectors as the direction angles for locating the injected sources.

\section{Modifications for the proposed technique}

According to the principle of the EIC of [4] as described in Section 2, we note that the resulting optimal weight vector given by $(10)$ is an $(M-q) \times 1$ vector. Let $\pi_{1}$ and $\pi_{n}$ denote the input power of the desired signal and the input noise power, respectively. Due to the loss of $q$ degrees of freedom, the maximum output SINR achieved by using the EIC of [4] is equal to $(M-q) \pi_{1} / \pi_{n}$ which is less than $M \pi_{1} / \pi_{n}$ achieved by using a conventional beamformer with array size $M$. Moreover, if the spatial smoothing approach like the one presented in [6] is used for dealing with the situation of coherent signal sources. Then, more degrees of freedom are lost. To avoid the problem of losing the degrees of freedom, we present modifications based on the use of the shift-invariant property of a ULA for enhancing the capabilities of the proposed technique.

First, consider the ideal situation where the steering angle $\theta_{0}$ equals the direction angle $\theta_{1}$ of the desired signal and $J=P-1$. Since the response of the $m$ th sensor to a signal with unit amplitude and a direction angle $\theta_{i}$ is given by $a_{m}\left(\theta_{i}\right)=\exp \left(\mathrm{j} 2 \pi(m-1) d \sin \left(\theta_{i}\right) / \lambda\right)$, we have the following relationship:

$$
\begin{gathered}
{\left[a_{m+1}\left(\theta_{2}\right), a_{m+1}\left(\theta_{3}\right), \ldots, a_{m+1}\left(\theta_{P}\right)\right]} \\
\quad=\left[a_{m}\left(\theta_{2}\right), a_{m}\left(\theta_{3}\right), \ldots, a_{m}\left(\theta_{P}\right)\right] \Phi_{j},
\end{gathered}
$$

due to the shift-invariant property possessed by a ULA, where the $J \times J$ diagonal matrix $\Phi_{j}$ is given by

$$
\begin{aligned}
\Phi_{j}=\operatorname{diag}\{ & \exp \left(\mathrm{j} 2 \pi d \sin \left(\theta_{2}\right) / \lambda\right), \\
& \exp \left(\mathrm{j} 2 \pi d \sin \left(\theta_{3}\right) / \lambda\right), \ldots, \\
& \left.\exp \left(\mathrm{j} 2 \pi d \sin \left(\theta_{P}\right) / \lambda\right)\right\} .
\end{aligned}
$$

Let the response matrix due to the interferers be denoted by $\boldsymbol{A}_{j}=\left[A\left(\theta_{2}\right), A\left(\theta_{3}\right), \ldots, A\left(\theta_{P}\right)\right]$. Two submatrices $\bar{A}_{j 1}$ and $\bar{A}_{j 2}$ of $A_{j}$ are constructed by letting $\bar{A}_{j 1}$ and $\bar{A}_{j 2}$ contain the first $J$ rows and the rows from the second row to the $(J+1)$ th row of $\boldsymbol{A}_{j}$, respectively. Thus, the matrix $\Phi_{j}$ shown in (22) can be obtained from $\bar{A}_{j 1}$ and $\bar{A}_{j 2}$ as follows:

$\Phi_{j}=\bar{A}_{j 1}^{-1} \bar{A}_{j 2}$. 
Using (22) and the matrix $\overline{A_{j}}$ as defined in (5), we can easily show that the response matrix $A_{j}$ associated with $X(t)$ is given by

$$
A_{j}=\left[\bar{A}_{j}^{\mathrm{T}},\left(\bar{A}_{j 3} \Phi_{j}\right)^{\mathrm{T}},\left(\bar{A}_{j 3} \Phi_{j}^{2}\right)^{\mathrm{T}}, \ldots,\left(\bar{A}_{j 3} \Phi_{j}^{q}\right)^{\mathrm{T}}\right]^{\mathrm{T}},
$$

where $\bar{A}_{j 3}$ represents the last row of $\bar{A}_{j}$. Although $\bar{A}_{j}$ is practically unknown, a basis matrix $\bar{E}_{j}$ of range $\left\{\bar{A}_{j}\right\}$ can be found by utilizing the procedure as described in Section 2. Similar to the construction of $\boldsymbol{A}_{j}$ as shown by (24), we can construct a matrix $E_{j}$ from $\bar{E}_{j}$ as follows:

$\boldsymbol{E}_{j}=\left[\overline{\boldsymbol{E}}_{j}^{\mathrm{T}},\left(\overline{\boldsymbol{E}}_{j 3} \Gamma_{j}\right)^{\mathrm{T}},\left(\overline{\boldsymbol{E}}_{j 3} \Gamma_{j}^{2}\right)^{\mathrm{T}}, \ldots,\left(\overline{\boldsymbol{E}}_{j 3} \Gamma_{j}^{q}\right)^{\mathrm{T}}\right]^{\mathrm{T}}$,

where $\overline{\boldsymbol{E}}_{j 3}$ represents the last row of $\overline{\boldsymbol{E}}_{j}$ and $\Gamma_{j}$ is a $J \times J$ matrix given by

$\Gamma_{j}=\bar{E}_{j 1}^{-1} \overline{\boldsymbol{E}}_{j 2}$

where $\overline{\boldsymbol{E}}_{j 1}$ and $\overline{\boldsymbol{E}}_{j 2}$ contain the first $J$ rows and the rows from the second row to the $(J+1)$ th row of $\bar{E}_{j}$, respectively. Based on the fact that range $\left\{\bar{E}_{j}\right\}=\operatorname{range}\left\{\bar{A}_{j}\right\}$ and the results given by (21)(24), we have the following theorem.

Theorem 1. It follows from (25) that range $\left\{\boldsymbol{E}_{j}\right\}=$ range $\left\{\boldsymbol{A}_{j}\right\}$.

Proof. Since range $\left\{\overline{\boldsymbol{E}}_{j}\right\}=\operatorname{range}\left\{\overline{\boldsymbol{A}}_{j}\right\}$, there exists a nonsingular matrix $T$ with size $J \times J$ such that $\bar{E}_{j}=$ $\overline{\boldsymbol{A}}_{j} \boldsymbol{T}$ and hence $\overline{\boldsymbol{E}}_{j k}=\overline{\boldsymbol{A}}_{j k} \boldsymbol{T}, k=1,2,3$. Consequently, we have from (26) that $\Gamma_{j}=T^{-1}\left(\bar{A}_{j 1}^{-1} \bar{A}_{j 2}\right) T=T^{-1}$ $\left(\Phi_{j}\right) \boldsymbol{T}$. Hence, $\bar{E}_{j 3} \Gamma_{j}^{r}=\bar{A}_{j 3} T\left(T^{-1} \Phi_{j} T\right)^{r}=\bar{A}_{j 3} \Phi_{j}^{r} T$ for $r=1,2, \ldots, q$. Therefore, it is easy to show from (25) that $\boldsymbol{E}_{j}$ can be expressed as $\boldsymbol{E}_{j}=\boldsymbol{A}_{j} \boldsymbol{T}$ and range $\left\{\boldsymbol{E}_{j}\right\}=$ range $\left\{\boldsymbol{A}_{j}\right\}$. This completes the proof.

Therefore, the lost degrees of freedom due to the signal blocking matrix $\boldsymbol{B}$ can be recovered.

Next, based on the above results, the capabilities of the proposed technique presented in Section 3 can be enhanced by incorporating the modifications. The criteria for determining the number and the powers of the injected sources are still the same as those described in Sections 3.1 and 3.2, respectively. However, the direction angles for locating the injected sources must be decided according to the following criterion due to the modifications.
Step 1. Construct the direction vectors $A\left(\alpha_{k}\right)$ with the direction angles $\alpha_{k}=\arcsin (2 k / M)$ for $k=-$ $\langle M / 2\rangle,-\langle M / 2\rangle+1, \ldots,-1,1,2, \ldots,\langle M / 2\rangle$ corrcsponding to the nulls of the quiescent beam pattern of the original $M$-sensor array, where $\langle x\rangle$ denotes the largest integer not greater than $x$.

Step 2. Compute the projection of each $A\left(\alpha_{k}\right)$ onto the correlation matrix $\boldsymbol{R}$ of the received data vector $X(t)$ as follows:

Projection $\left\{A\left(\alpha_{k}\right)\right\}=A^{\mathrm{H}}\left(\alpha_{k}\right) R A\left(\alpha_{k}\right)$.

Step 3. Choose the $\Delta_{1}+\Delta_{2}$ smallest projections from all of the projections computed from (27) and their corresponding direction vectors.

Step 4. Take the direction angles associated with these $\Delta_{1}+\Delta_{2}$ direction vectors as the direction angles for locating the injected sources.

The proposed technique with modifications in the case of using a ULA is summarized as follows. We first construct the correlation matrix $\overline{\boldsymbol{R}}_{\mathrm{a}}$ based on artificial source injection using (14) with the modifications in determining the direction angles for locating the injected sources described as above and then perform the GEVD of $\overline{\boldsymbol{R}}_{\mathrm{a}}$ to obtain the basis matrix $\overline{\boldsymbol{E}}_{j}$. By using (25), the basis matrix $\boldsymbol{E}_{j}$ for the IS spanned by the received data vector $X(t)$ is computed. After finding $E_{j}$, the optimal weight vector without loss of degrees of freedom can be computed by solving the following optimization problem:

Minimize $W^{\mathrm{H}} W$

$$
\text { subject to } W^{\mathrm{H}} \boldsymbol{E}_{j}=0 \text { and } W^{\mathrm{H}} A\left(\theta_{0}\right)=1 \text {. }
$$

The optimal solution for (28) is given by

$W_{0}=\kappa_{1}\left(\boldsymbol{I}-\boldsymbol{E}_{j}\left(\boldsymbol{E}_{j}^{\mathrm{H}} \boldsymbol{E}_{j}\right)^{-1} \boldsymbol{E}_{j}^{\mathrm{H}}\right) A\left(\theta_{0}\right)$,

where $\kappa_{1}$ represents the corresponding normalization constant.

\section{Simulation examples and comparison}

In this section, several simulation examples for illustration and comparison are presented. In order to concentrate on the results due to the steering angle error, ensemble correlation matrices required by each example are used. The adaptive array considered for all simulations is a 12-sensor ULA with 
interelement spacing equal to $\lambda / 2$. Assume that the sensor noise is spatially white with unit power. Let the steering angle be equal to zero degree and the direction angle of the desired signal be equal to zero degree for correct steering and two degrees off broadside for incorrect steering. The input SNR is equal to $3 \mathrm{~dB}$. Two interferers with $\mathrm{INR}=10 \mathrm{~dB}$ are impinging on the array from $-32^{\circ}$ and $-36^{\circ}$ off broadside. The correlation coefficients between these three partially correlated signal sources are given by $\rho_{12}=0.2 \exp (0.5 j), \rho_{13}=0.2 \exp (0.9 j)$ and $\rho_{23}=0.2 \exp (0.1 j)$ for the signal pairs $\left(s_{1}(t), s_{2}(t)\right)$, $\left(s_{1}(t), s_{3}(t)\right)$ and $\left(s_{2}(t), s_{3}(t)\right)$, respectively.

Example 1. Here, we illustrate the performance deterioration of the EIC of [4] in the presence of steering angle error and overestimation of the interference number. A blocking matrix $\boldsymbol{B}$ with $q=1$ as shown in (4) is used for this example. Fig. 1(a) shows the output beam patterns of the EIC without steering angle error. Comparing the performances of the EIC for the circumstances where the estimated numbers of the interferers are equal to two and three, respectively, we observe that overestimation of the interference number causes almost no effect on interference suppression. However, it leads to reducing the capability of noise

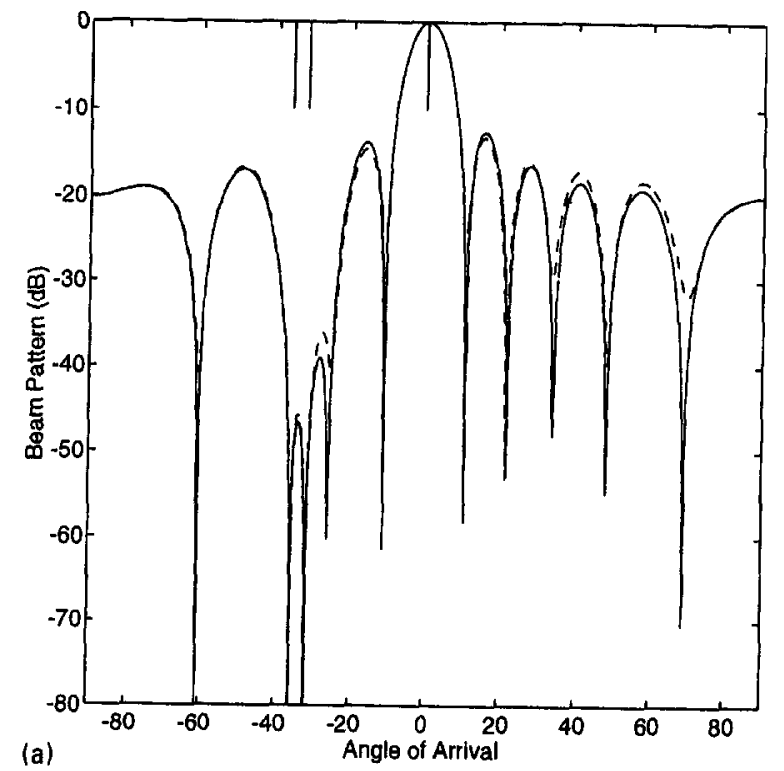

suppression due to one additional constraint induced by the overestimation of the interference number.

Fig. 1(b) plots the beam patterns for the EIC with steering angle error. In this case, we note that overestimation of the interference number results in very serious performance degradation. The desired signal is viewed as an interferer and almost rejected regardless of the input SNR. In contrast, the desired signal suffers only little attenuation when the number of the interferers is estimated correctly. This is because $\left|v_{1}\right|^{2} \pi_{1} \ll\left|v_{i}\right|^{2} \pi_{i}$, for $i=2,3$ and thus the first two principal $g$-vectors obtained from the GEVD of the correlation matrix $\overline{\boldsymbol{R}}$ contain almost no information about the desired signal. Nevertheless, there exists difference between the estimated IS and the actual IS due to the leakage of the desired signal in the blocked data vector. Hence, the EIC cannot provide sufficient interference suppression.

Example 2. This example illustrates the robust capabilities of the EIC using the proposed technique presented in Section 3. Let the estimated interference number $\tilde{J}$ be 2 and $\Delta_{1}=\Delta_{2}=1$. Again, a blocking matrix $B$ with $q=1$ as shown in (4) is used and the steering angle error is present. According to the criterion described in Section 3.1, we use two injected

Fig. 1. The result of Example 1. Solid line: correct estimation of the interference number. Dash line: overestimation of the interference number. (a) The output beam patterns without steering angle error. (b) The output beam patterns with steering angle error. 

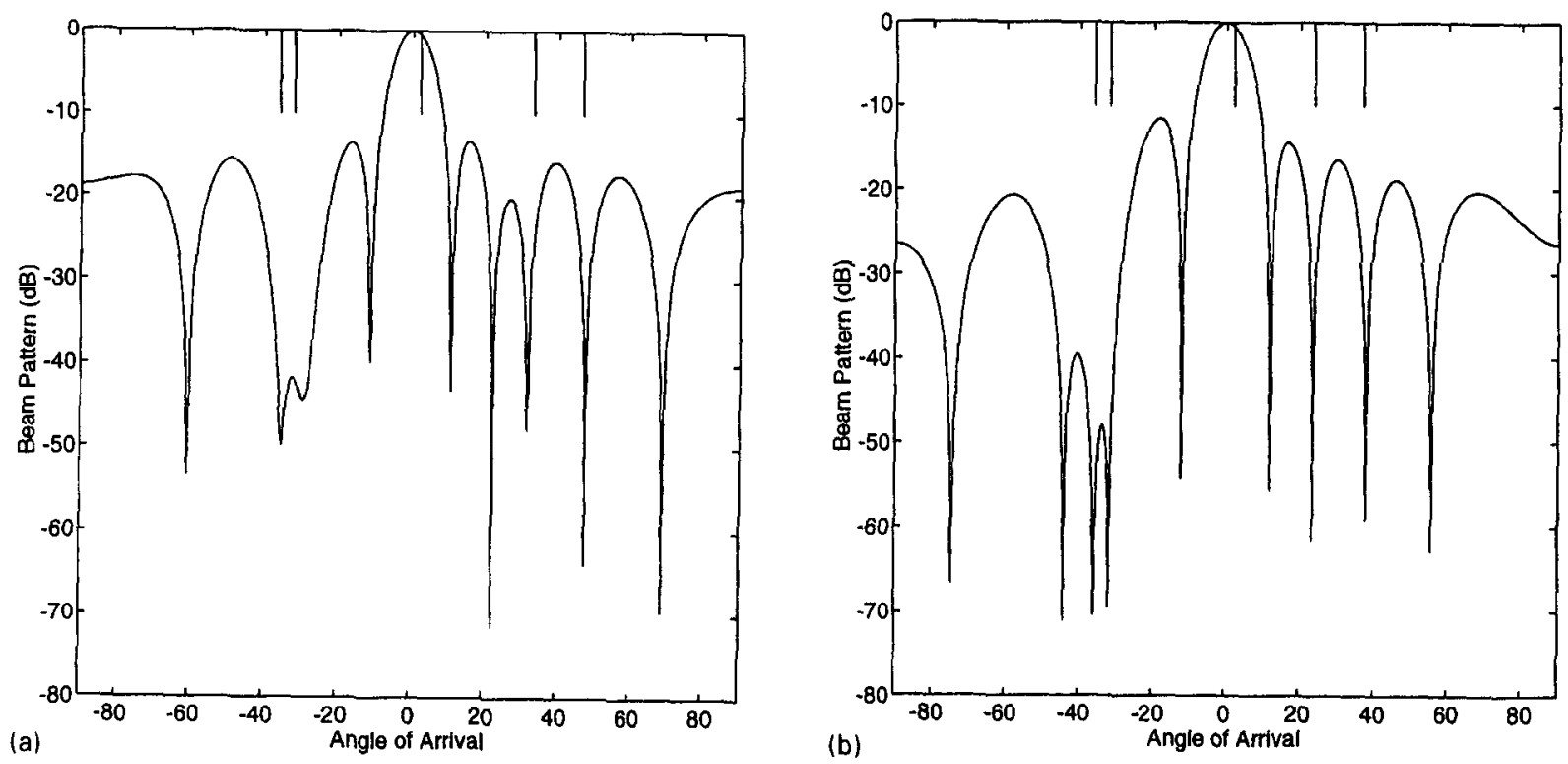

Fig. 2. The result of Example 2. The output beam patterns of the proposed technique. (a) Using a blocking matrix with $q=1$. (b) Using a blocking matrix with $q=2$.
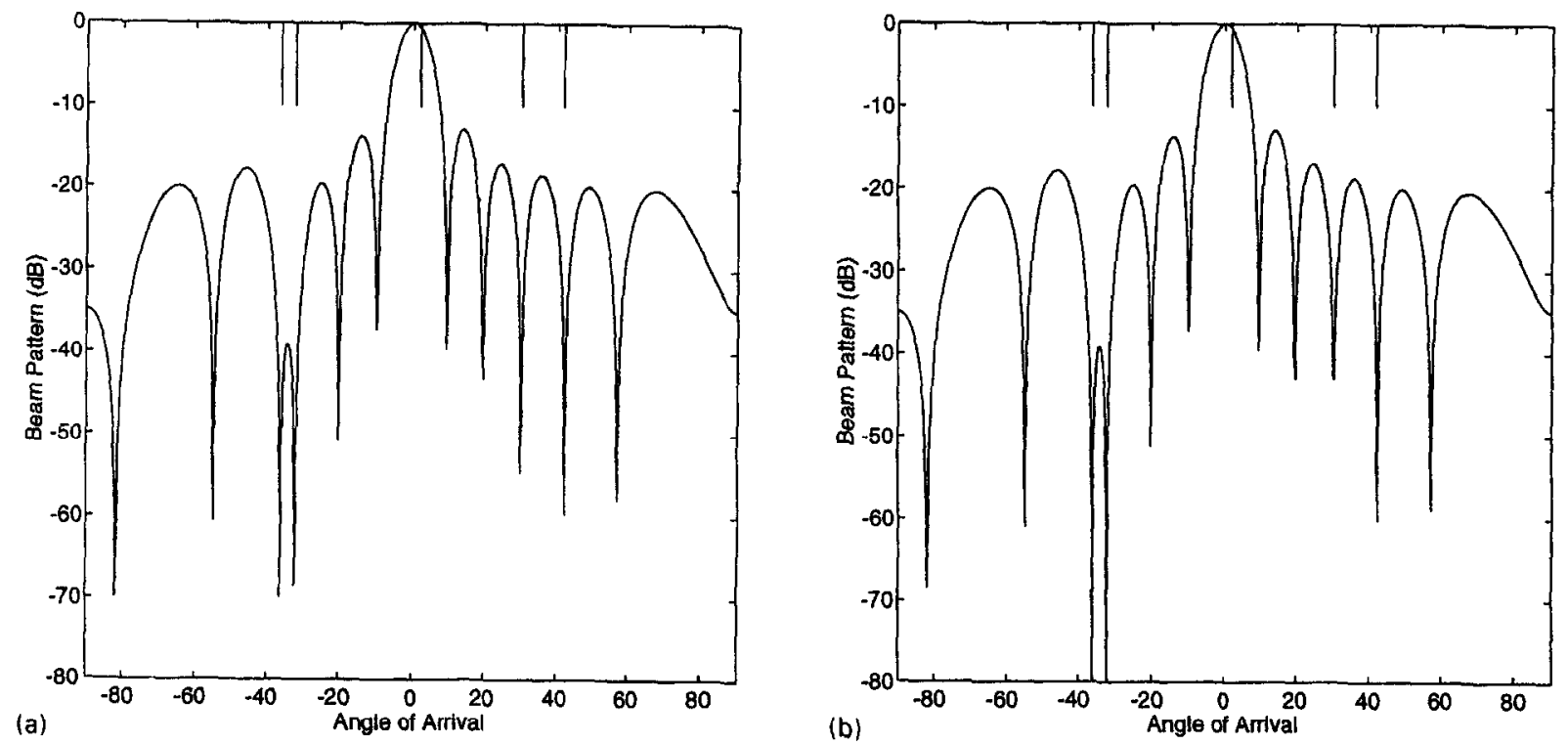

Fig. 3. The result of Example 3. The output beam patterns of the proposed technique with modifications. (a) Using a blocking matrix with $q=2$. (b) Using a blocking matrix with $q=3$. 
sources with SNR $=0 \mathrm{~dB}$ and set the dimension $J$ of the IS equal to 3 . Their direction angles are set to $\arcsin (2 k / M-1)$ for $k=3,4$, i.e., $\arcsin (6 / 11)$ and $\arcsin (8 / 11)$, respectively, according to the criterion described in Section 3.2. Fig. 2(a) depicts the resulting beam pattern. Comparing the results of Figs. 1(b) and 2(a), we can see that the proposed technique is effective for dealing with the situation where both of the steering angle error and overestimation of interference number are encountered.

Next, we consider the use of a blocking matrix with higher order. Let the order of the blocking matrix be $q=2$. This will lose one additional degree of freedom. Hence, the direction angles of the two injected sources are changed to $\arcsin (2 k / M-2)$ for $k=2,3$, i.e., $\arcsin (2 / 5)$ and $\arcsin (3 / 5)$, respectively, according to the criterion described in Section 3.2. The simulation result is shown in Fig. 2(b). The corresponding beam pattern possesses decper nulls in the interference directions but with wider mainlobe as compared with that of Fig. 2(a).

Example 3. As shown in Example 2, using a blocking matrix with higher order produces more interference suppression at the price of wider mainlobe due to more degrees of freedom lost. Here, we illustrate the effectiveness of the proposed technique in conjunction with the modifications presented in Section 4. All of the signal characteristics are the same as those given in Example 2 cxcept that the direction angles of the two injected sources are set to $\arcsin (2 k / M)$ for $k=3,4$, i.e., $\arcsin (1 / 2)$ and $\arcsin (2 / 3)$, respectively, according to the criterion described in Section 4 . The beam patterns of using a blocking matrix with $q=2$ and $q=3$ are shown in Figs. 3(a) and (b), respectively. The array gains at the interference direction angles of $-32^{\circ}$ and $-36^{\circ}$ are -68.434 and $-69.780 \mathrm{~dB}$, respectively, for Fig. 3(a), while they are -91.348 and $-82.220 \mathrm{~dB}$, respectively, for Fig. 3(b). From the simulation results, we observe that the widths for the mainlobe are almost the same for these two beam patterns and are both narrower than those shown in Fig. 2. Moreover, Fig. 3(b) demonstrates that the EIC using a blocking matrix with higher order provides more interference suppression because the difference between the estimated IS and the actual IS is reduced.

\section{Conclusion}

This paper has presented an efficient technique for the maximum rejection of interference when using an eigenanalysis interference canceller (EIC). In the presence of steering angle error, we have proposed to prevent the response vector of the desired signal from lying in the resulting interference subspace by imposing artificial signal sources. The criteria required for determining the characteristics including the number, the direction angles and the powers of the artificial signal sources have been presented. Modifications for enhancing the proposed technique have also been presented to alleviate the drawback like the loss of degrees of freedom based on the shift-invariant property of a uniform linear array. It has been shown that the proposed technique makes the EIC possess robust capabilities against steering angle error. Computer simulations have demonstrated the effectiveness of the proposed technique.

\section{References}

[1] M.G. Amin, Concurrent nulling and locations of multiple interference in adaptive antenna arrays, IEEE Trans. Signal Process. 40 (1992) 2658-2663.

[2] Y. Bresseler, V.U. Reddy, T. Kailath, Optimum beamforming for coherent signals and interference, IEEE Trans. Acoust. Speech Signal Process. ASSP-36 (1988) 833-842.

[3] B. Friedlander, A signal subspace method for adaptive interference cancellation, IEEE Trans. Acoust. Speech Signal Process. ASSP-36 (1988) 1835-1845.

[4] A.M. Haimovich, Y. Bar-Ness, An eigenanalysis interference canceller, IEEE Trans. Signal Process. 39 (1991) 76-84.

[5] K. Ohishi, R.T. Milton, A new optimization technique for adaptive antenna arrays, IEEE Trans. Antennas Propagation AP-41 (1993) 525-532.

[6] T.-J. Shan, T. Kailath, Adaptive beamforming for coherent signals and interference, IEEE Trans. Acoust. Speech Signal Process. ASSP-33 (1985) 527-536.

[7] H. Subbaram, K. Abend, Interference suppression via orthogonal projections: A performance analysis, IEEE Trans. Antennas Propagation AP-41 (1993) 1187-1193.

[8] M. Wax, T. Kailath, Detection of signals by information theoretic criteria, IEEE Trans. Acoust. Speech Signal Process. ASSP-33 (1985) 387-392.

[9] Q. Zhang, K.M. Wong, P.C. Yip, J.P. Reilly, Statistical analysis of the performance of information theoretic criteria in the detection of the number of signals in array processing, IEEE Trans. Acoust. Speech Signal Process. ASSP-36 (1988) 15571566. 